\title{
Una verdad estética para la teoria de la historia
}

\author{
An aesthetic truth for the theory of history
}

\author{
EUGENIA GAY \\ Escuela de Historia, \\ Facultad de Filosofia y Humanidades, \\ Universidad Nacional de Córdoba, \\ Centro de Historia Intelectual \\ Universidad Nacional de Quilmes (Argentina) \\ eugeniagay@gmail.com
}

RESUMEN

En este ensayo intentamos proporcionar una definición de la teoria de la historia que contemple su función como ordenadora y productora de la tradición historiográfica. Mediante este procedimiento proponemos que, dado el fin de la certeza unánime sobre la conveniencia del paradigma científico para el conocimiento histórico, es necesario rediscutir el significado del concepto de verdad que lo subyace. Tal redefinición se impone debido al carácter identitario que el compromiso con la verdad comporta para la historiografia, pero resulta incomprensible desde el interior del paradigma epistemológico. En el sentido de dar un nuevo inicio a ese debate, proponemos la aproximación estética de la hermenéutica filosófica como alternativa a la noción de verdad científica de la epistemología.

Palabras clave: Teoria de la historia; verdad histórica; estética.

ABSTRACT

In this essay we try to provide a definition of the theory of history that contemplates its function as organizer and producer of the historiographical tradition. Through this procedure we propose that, given the end of unanimous certainty about the convenience of the scientific paradigm for historical knowledge, it is necessary to rediscuss the meaning of the concept of truth that underlies it. Such a redefinition is imperative due to the identity forming character that the commitment to truth entails for historiography, but cannot be understood from within the epistemological paradigm. With the purpose of giving a new inauguration to this debate, we propose the aesthetic approach of philosophical hermeneutics as an alternative to the notion of scientific truth of epistemology.

Esta obra está sujeta a la Licencia Reconocimiento-NoComercial-CompartirIgual 4.0 Internacional de Creative Commons. http://creativecommons.org/licenses/by-nc-sa/4.0/ 
Keywords: Theory of history; historical truth; aesthetics.

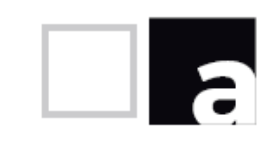

El mundo en el que Marc Bloch (1996) escribió su apología de la historia ha desaparecido. La idea romántica y tranquilizadora de que el historiador, armado de sus cuadernos y sus lápices, podría dirigirse al archivo para encontrar los documentos con los cuales responder sus inquietudes se ha transformado en una ficción tan agradable como inexistente ${ }^{1}$. La expectativa de ver la compleción de los archivos en algún tiempo más lejano o más cercano se ha esfumado ${ }^{2}$ y la posibilidad de un futuro hipotético en el que el

\footnotetext{
1 Aquí la palabra "ficción" se utiliza en sus múltiples sentidos, para recordar que el golpe de gracia a esta ilusión entre los historiadores debe atribuirse a Hayden White y su decisivo Metahistoria, (1973) en el cual, entre otras cosas, se desmonta la necesidad de una correspondencia entre lo narrado y lo sucedido, la cual se desprende por ejemplo, de la premisa básica sobre la que asienta su trabajo: "consideraré al trabajo histórico como lo que es más manifiestamente - esto es, una estructura verbal en forma de un discurso de prosa narrativa que pretende ser un modelo, o ícono, de estructuras y procesos pasados con el objetivo de explicar lo que fueron mediante su representación"(p.2) de la siguiente afirmación que se refiere a los clásicos historiográficos del siglo XIX: "Su status como posibles modelos de representación o conceptualización histórica no depende de la naturaleza de "los datos" que utilizaron para fundamentar sus generalizaciones o las teorias que invocaron para explicarlas; depende más bien de la consistencia, coherencia y poder iluminador de sus respectivas visiones del campo histórico" (p. 4). Este planteamiento del trabajo provocó un inicial rechazo generalizado que mostraba la magnitud del cimbronazo dentro de la disciplina, cuyas consecuencias pueden compararse con los efectos de la Reforma luterana en el catolicismo: separar lo representado de aquello que se representa equivalía a poner en duda la autoridad de la Iglesia como interventora entre el hombre común y su Dios, desatando una tormenta similar de cuestionamientos internos y externos que transformaron los presupuestos fundamentales del mundo, en este caso de los historiadores. Para dimensionar la transformación, podemos notar por ejemplo que en Meaning, Truth, and Reference in Historical Representation. (Ithaca: Cornell University Press) Frank Ankersmit (2012) asume ya como un dato la división entre "escritura histórica' (Geschichtsschreibung) e 'investigación histórica' (Geschichtsforschung). Si bien la crítica al modelo Hempeliano (Hempel, 1942, pp. 35-48.) comenzara casi en simultáneo con su proposición (ver por ejemplo, Dray, 1957.), la idea de correspondencia entre representación y referente permaneció como base, a veces inconfesada, de una deseada cientificidad de la verdad histórica. Para una defensa de las leyes históricas véase, por ejemplo, Leuridan y Froeyman (2012: 172-92). Para una perspectiva más amplia, véase Misak (1995).

2 La idea de compleción es una de las bases de la historiografia decimonónica, apoyada sobre todo en la filosofia de la historia mejor expresada por Hegel (2005), la cual no debe ser separada de su idea general del movimiento del espiritu hacia una mayor claridad (en rigor esta idea se desarrolla en la Filosofía del Espíritu), que se alcanzaría por medio de una acumulación dialéctica. Aunque esta proposición haya sido muchas veces apropiada de forma esquemática y un tanto empobrecida por parte de los historiadores, la concepción según la cual la filosofia es el camino hacia el concepto ha servido de base para sostener la provisionalidad del conocimiento histórico en un esfuerzo conjunto de constituir finalmente la totalidad de la historia por vía de agregación: de esa premisa depende la confianza en el progresivo avance del conocimiento apoyado en la paulatina revelación de nuevos documentos y en la creciente
} 
papel desaparece y vastas secciones del pasado quedan huérfanas de documentación es hoy una realidad plausible. Junto con la idea del historiador erudito de la Europa de los años cuarenta, se ha esfumado también la expectativa, contenida en los escritos del eminente medievalista, de que la sociedad se orientaba inexorablemente hacia una organización cada vez más ajustada no sólo de sus archivos, sino también de su presente. La vieja idea del progreso, con la que durante tanto tiempo discutimos, la idea de que evolucionamos hacia un futuro cada vez mejor (mejor organizado, mejor distribuido, mejor documentado) parece hoy en dia una antigüedad ${ }^{3}, \operatorname{como}$ las pirámides o las batallas de elefantes. Se trata de la debacle de toda una constelación de significados que exceden a la producción de conocimiento, involucrando a la totalidad del entramado social. Como el propio Bloch, lo que entendiamos por historia, en su doble aspecto de démarche de la humanidad y de registro ${ }^{4}$, sucumbió a la maldad del siglo XX en las mazmorras despiadadas de regimenes totalitarios 5 .

La descomunal diversidad de los documentos necesarios para componer cualquier historia en nuestra era es sólo comparable a la diversidad de temas que la historiografia explora y a la complejidad del mundo en que vivimos ${ }^{6}$, en términos de comunicaciones, de relaciones, de instituciones e identidades. Probablemente, la verdad no sea que nuestros testimonios, nuestro sistema jurídico y nuestros periódicos estén hoy más dedicados al engaño y la tergiversación que los de antaño, sino que hoy en día sospechamos más, somos más vigilantes de los intereses que llevan a las personas a realizar declaraciones en uno $u$ otro sentido, de la escandalosa manipulación de la justicia, las estadísticas y las declaraciones de impuestos e incluso de los

habilidad de los historiadores para descifrar manuscritos o jeroglíficos, en el desarollo de nuevas técnicas de datación, de medición y de interpretación, los cuales justificarian la afirmación de que "cada vez conocemos mejor", como sostiene por ejemplo la escuela que sigue los pasos de Lucien Febvre (véase Braudel, 1970) entre tantos otros. Es esa concepción la que se encuentra asimismo en el conocido debate sobre la secularización, cuyas figuras destacadas son Hans Blumenberg y Karl Löwith (ver para este tema el resumen de la controversia realizado en: Wallace (1981: 63-79).

3 Ya en la obertura de su retrospectiva de 1920 señalaba el teórico de Cambridge J.B. Bury (1920) que la idea de Progreso se había transformado en un idolum saeculi por lo menos discutible, y sin dudas reducida a cuestión de fe. Para que no quepan dudas, aclararemos que la idea de progreso y la concepción de la compleción del conocimiento son nociones que se complementan mutuamente y que no pueden funcionar una independientemente de la otra. Por lo tanto, vale la nota anterior como complemento de esta otra.

${ }^{4}$ Reinhart Koselleck $(1994 ; 2010)$ ha descripto el colapso de los dos significados de la palabra historia (Geschichte) para el universo de la lengua alemana, pero su tesis pretende ser y ha sido extrapolada a la totalidad de la historia moderna.

5 Se ha señalado que uno de los eventos históricos que más han contribuido a dar por tierra con la idea del progreso de la ciencia ha sido la Segunda Guerra Mundial, en especial por el uso que el nazismo hizo de la técnica y por el desastre de la bomba nuclear.

6 Una buena crónica de los azares que la exploración archivística depara a cualquier investigador es el libro de Lila Caimari (2017). 
trabajos pretensamente científicos. Y esto no significa un quiebre entre la historia que se escribe sobre épocas anteriores y la historia que escribimos sobre épocas más recientes, sino - una vez más - la completa reformulación de los presupuestos que sostienen el conjunto de nuestras investigaciones. Pretender o suponer la honestidad de los testimonios o de las "huellas", o incluso la capacidad de identificar fidedignamente las impostaciones como una de las bases de la historia científica ${ }^{7}$ es, por lo menos, ingenuo. Ni hablar de lo que hoy podríamos llamar "documentos personales", en tiempos en que las personas ya no escriben cartas, sino e-mails o, peor aun, mensajes de texto; ya no escriben en sus diarios, sino en un "blog" o en su "muro de Facebook", o Twitter, o Instagram. La propia politica, antes tan oficial y burocrática, puede ser hoy modificada radicalmente por un comentario en una red social. No existen repositorios capaces de contener esta clase de información, ni criterios que puedan garantizar la veracidad de los documentos, y ni siquiera de las estadísticas. ¿Significa esto que debemos abandonar la pretensión de realizar una historia que los incorpore? ¿Qué ha quedado entonces, del "oficio del historiador" elogiado por Bloch? ¿Podemos pensar una historiografia que desista de su compromiso, sin importar cuán romántico, con la verdad? A pesar de las consideraciones del narrativismo y el posnarrativismo que así lo consideran, ${ }^{8}$ entiendo que no. Que el compromiso de los historiadores con alguna clase de verdad pertenece al orden de lo identitario9. Tal vez debamos, en cambio, volver a discutir qué clase de verdad esperamos encontrar.

La teoria de la historiografia es el terreno donde se cuestionan las verdades asumidas de la historiografia, donde se discuten las visiones del mundo según las cuales los historiadores realizan sus trabajos, y la validez de los métodos y presuposiciones conceptuales que rigen el oficio del historiador en su faceta más técnica. Esto se muestra en forma de discusiones sobre el carácter del

\footnotetext{
7 Este era el presupuesto sobre el que se fundara la "microhistoria" del afamado Carlo Ginzburg (1981), y que se expresa en el prefacio de su libro canónico: es posible leer un documento a pesar de sus intenciones.

8 Esta es la propuesta que guía, por ejemplo, a Kuukkanen (2015).

9 Una discusión sobre la relación entre la "compleción" o la "unidad" y la verdad se encuentra en Ricoeur (1990). Como veremos más adelante, la idea de verdad científica moderna adoptada por la historiografia postula la idea de correspondencia "La definición nominal de la verdad, a saber: que es la coincidencia del conocimiento con su objeto, se concede aquí y se presupone." (Kant, 2009, p. 128). En los tiempos actuales esa relación ya no debe expresarse entre la interpretación del historiador y los "hechos" (unidad desmontada por el narrativismo), sino entre las afirmaciones de los historiadores y los documentos, los cuales componen el "aparato crítico" del texto científico. El historicismo identificó a la verdad con la historia, como se lee en Herder (1877): "Was ich bin, bin ich geworden. Wie ein Baum bin ich gewachsen; der Keim war da; aber Luft, Erde und alle Elemente, die ich um mich setze, mussten beitragen, den Keim, die Frucht, den Baum zu bilden." Incluso las teorias más radicalmente antirrepresentacionistas asumen este compromiso con alguna clase de verdad (Ankersmit, 1996). El escándalo en este último caso parece provenir de la atribución de verdad también a la literatura. En cualquier caso, hago mías las palabras de Ankersmit en ese texto: "No deberíamos preguntarnos cómo difieren la historia y la literatura desde la perspectiva de alguna noción de verdad dada a priori, sino cómo se manifiesta la verdad en la historia y la literatura respectivamente, partiendo del supuesto de que cada una ejemplifica una forma específica de verdad" (p. 149).
}

\section{anuario.}


tiempo y sobre las diferentes concepciones de la memoria; en disquisiciones sobre el carácter de la verdad que la historiografia es capaz de producir y sobre la realidad del pasado, en las que se cuestiona, por ejemplo, la opacidad de los mecanismos por los cuales se conforman y se exploran los repositorios. En cualquier trabajo de investigación, la reflexión teórica no puede limitarse a la selección de conceptos aislados o teorías completas a ser aplicadas sobre un territorio nuevo o sobre un conjunto inexplorado de fuentes. Concebido como un esfuerzo de investigación dotado de originalidad, un trabajo de interpretación histórica implica la creación de conceptos y categorías que den forma a una temática de modo que ésta pueda ser captada, percibida y discutida por la comunidad intelectual y el conjunto de la sociedad. El trabajo del historiador no se limita a la descripción y reunión de acontecimientos, sino que siempre pretende dar un sentido a la realidad y un sentido de la realidad.

Sin embargo, existe un cisma entre las discusiones actuales, más o menos radicalizadas, en el campo de la teoría de la historia y la práctica efectiva de la escritura de la historia, que se sostiene sobre una supuesta autonomía de la práctica con respecto a la teoría. El riesgo de esta distancia es que la discusión sobre la historiografía vuelva a colocarse, como antaño, en el lugar opuesto a la práctica, creando una disociación en lo que debería ser una tarea conjunta de reflexión sobre el trabajo del historiador ${ }^{10}$.

Considerando este panorama, quisiera proponer una reflexión sobre la relación de la teoria de la historiografia con la práctica de la historiografia, que se articula sobre la idea de que la búsqueda de la verdad representa, como ya hemos señalado, un rasgo identitario de la historiografia. Esto implicará, principalmente, una redefinición del concepto de verdad que pueda zurcir nuestras discusiones teóricas con aquello que resulta de nuestra práctica. Propondré, como estrategia, considerar tres dimensiones de la teoría de la historia, de acuerdo a las cuales podemos organizar el pensamiento para escapar a las nomenclaturas tradicionales de teoría, metodología, taller del historiador, etc. Estas dimensiones se pueden enunciar de la siguiente manera: la teoría de la historia es algo que sabemos; es algo que hacemos y es algo que sucede.

\section{La teoria de la historia es algo que sabemos.}

\footnotetext{
10 Esta división se expresa, por ejemplo, en el ya citado Metahistory de Hayden White (1973), en el cual se separa el trabajo de los "historiadores propiamente dichos" del trabajo de los "filósofos de la historia", una división que también encuentra expresión en el clásico de Benedetto Croce (1921), no menos que en la conocida controversia entre Louis Althusser y E.P. Thompson (Rendueles, 2013, pp.177-97).
} 
Desde sus orígenes griegos, los historiadores han tratado de definir lo que hacen de acuerdo con ciertos parámetros, que diferencian su esfuerzo de otras actividades de producción de significado. Heródoto lo hizo, y también Tucídides. La historia consistía entonces en permitir que los líderes y los pueblos aprendieran de sus errores y victorias pasadas, evitar que las grandes hazañas de la humanidad cayeran en el olvido, y enseñar moral y ética a las generaciones venideras. Cicerón ponderó a la historia como magistra vitae, Nicolás Maquiavelo como medio para elevar a la ciudad y Francesco Guicciardini como garante del verdadero poder político.

Durante los siglos XVIII y XIX, cuando la historia se construyó como una disciplina científica, historiadores y filósofos como Giambattista Vico, Johann Gottfried Herder, y Leopold von Ranke, intentaron definir los rasgos esenciales de la disciplina para ordenarla dentro del conjunto de las disciplinas científicas que disputaban, entonces, su autoridad. Definieron sus métodos y procedimientos, describieron el tipo de conocimiento que podría producir y la verdad que podriamos esperar de ella. Afinaron el apartamiento de la historia respecto del resto de las humanidades, definiéndola como la disciplina que se ocupa con el mundo "real", aquella que posee un mayor compromiso con el progreso y la razón de la humanidad ${ }^{11}$. Se establecieron temas y objetos de la historia, así como las líneas generales a lo largo de las cuales debía desarrollarse. En ese sentido, estos intelectuales también establecieron el propósito de la historiografía en línea con otras disciplinas de conocimiento práctico para el mundo real, como la política y la economía, mediante las cuales los Estados podian imponer la voluntad de poblaciones enteras, supuestamente en interés del progreso. La historia quedaba así firmemente atada a la construcción de la comunidad nacional12.

El siglo XX vio las redefiniciones más vertiginosas e imprevisibles para la historia. A principios de siglo, la historiografia todavía se concebía como el medio principal para construir una identidad común a partir de la cual las estructuras gubernamentales podrian cimentar su autoridad y construir consenso. Pero también fue tomada por diversas minorias como un instrumento para afirmar su independencia de tales órdenes, o su voluntad de cambiar el orden establecido, de barajar y dar de nuevo. Trabajadores, mujeres, pueblos colonizados produjeron nuevas concepciones de la marcha de la historia, que no solo los incluían, sino que también los colocaban en una posición clara dentro de la disputa por el poder ${ }^{13}$. Desde esas periferias, se

\footnotetext{
11 Véase, por ejemplo, Ranke (1979, pp. 509-517); Herder (2007, pp. 51-63; Vico), Giambatista (1995, pp. 158; 168; 170, etc.).

12 Sería ocioso reponer la discusión sobre la asociación entre historicismo y nacionalismo. Baste como referencia para profundizar en el tema a partir de su vasto conjunto de referencias, el libro de Beiser (2011).

13 Para las transformaciones de la historiografia en el siglo XX y la diversificación y apropiación de los discursos históricos por diferentes grupos de interés, sigue siendo canónico el texto de
}

\section{anuario.}


cuestionó la metodología de la historiografia y sus fuentes privilegiadas, y la narrativa tradicional, producida desde la perspectiva del vencedor, o del centro, o desde arriba, lo cual forzó a una reescritura casi completa del pasado.

Y después de las dos grandes guerras, la historia, sin importar cuán criticada y sospechosa, fue nuevamente convocada para reconstruir el consenso social necesario para vencer el terrible golpe asestado a la humanidad por su propia atrocidad. Una vez más se redefinió en forma y propósito, para enfrentar el nuevo desafio: el de comprender un mundo cada vez más intrincado y peligroso, en el que actuaría ya no como maestra, sino como juez del carácter y la moral. Para hacer esto, se instó a los historiadores a despojarse de su partidismo, a evaluar los asuntos desde el templo sagrado de la objetividad y a atenerse a los hechos. La asepsia en la recopilación de pruebas y la compostura en la interpretación se establecieron como base teórica de una reparación justa de daños a escala global, y las universidades se erigieron como vigilantes de afirmaciones inconsistentes. Mediante ese movimiento, las narrativas producidas fuera de los círculos académicos (por sobrevivientes, perpetradores o víctimas) fueron relegadas como una forma menor de comprensión, menos sofisticada y más peligrosa, clasificada bajo la rúbrica de "memoria"14 y, por lo tanto, considerada como un conocimiento subjetivo e inapropiado para la tarea, que en el mejor de los casos podría ser utilizada como fuente para un conocimiento que la profesión historiográfica pudiera considerar como válido. Lo cual resulta tanto más significativo si pensamos que historiadores que escribian solo algunos años antes, como el propio Marc Bloch (1996), de hecho utilizaban "historia" y "memoria" casi como sinónimos intercambiables.

Esta apuesta por la reconstrucción de un mundo capaz de sanar sus heridas y aventurarse a un futuro común resultó en una conservación del sacudido paradigma científico, y coincidió, como en muchos otros momentos de la historia de la historiografia, con la política de la construcción de la identidad nacional ${ }^{15}$. La redefinición de las formas y las metas del esfuerzo de conocimiento de la historiografía en la segunda mitad del siglo XX participó plenamente del espectro de sentidos con que se trabajó por la reconstrucción de los lazos sociales violentamente destruidos por guerras civiles y regimenes totalitarios, mediante la ponderación de la democracia liberal como principal

Iggers (2012). Véase también la idea de Estudios subalternos, en Raka Ray (2010). Para el aporte feminista a la historiografia véase Scott (2010, pp.221-244).

14 Sobre el "memory boom", ver por ejemplo, Tilmans y Winter (2010); Assmann (2006, pp. 261273); Jelin (2003). Estos textos son solo algunos de los que se han ocupado del tema. La bibliografia es extensa y en cierta forma particular a cada caso. Para una discusión del tema del testimonio ver: Mudrovcic (2007, pp. 127-50).

15 Sobre este tema ver Berger y Lorenz (2010). 
ordenador ${ }^{16}$. En otras palabras, el proyecto científico y el proyecto liberal pertenecen al mismo universo ideológico y pragmático, y se cimientan mutuamente.

El fundamento de la idea de progreso que subyace tanto al proyecto político como al proyecto de conocimiento actuales reside en dos presupuestos temporales esenciales: por un lado, la idea de un pasado terminado, que puede ser evaluado y juzgado y, por otro, la ponderación del futuro abierto e indeterminado como aquello que completa las "fallas" o ausencias del presente. La ruptura entre el espacio de experiencia y el horizonte de expectativa que Reinhart Koselleck (1993) identificara en el famoso Sattelzeit del siglo XIX europeo y cuyo rendimiento cautiva hoy a una porción nada despreciable de historiadores, pertenece más bien al momento de reconstrucción en que el historiador escribiera sus interpretaciones y es, más que una descripción del mundo, primordialmente la revalorización de un proyecto. En otros términos, la idea de romper con un pasado que pasa al terreno de lo ajeno y contar con un futuro tan extendido como indeterminado, responde más a la descripción del proyecto politico que a la descripción por así decir "factual" de la Modernidad". El conocimiento actual, que sigue alentando activamente ese proyecto, continúa presentándose como un conocimiento que se asume humildemente parcial o defectuoso, y cuya consumación se encuentra en un futuro que promete mejores instrumentos de observación y medición, y mejores técnicas de extracción y conservación. De ese modo la compleción, esto es, la "totalidad" o "universalidad" necesaria al paradigma científico en que se reconoce la historiografia, se concreta en un acto tan imaginativo como proléptico. La vinculación entre nuestra concepción del conocimiento y el proyecto liberal ${ }^{18}$ aparece también aquí: la promesa del capitalismo globalizado, expresión contemporánea del liberalismo, se articula sobre la necesidad de ahorrar hoy para prosperar mañana. De ese modo, la "ganancia" obtenida mediante las privaciones impuestas siempre resulta postergada ${ }^{19}$ : la compleción del proyecto liberal siempre se ubica en su proyección. El futuro debe permanecer abierto e indeterminado. De la misma manera, la verdad total de la historia reside en la agregación acumulativa, futura, de cada vez más puntos de vista. Es así que podemos afirmar, como

\footnotetext{
16 El presidente Raúl Alfonsín, por ejemplo, en su asunción tras las primeras elecciones libres en Argentina después de la dictadura militar afirmaba, "con la democracia no sólo se vota, sino que también se come, se educa y se cura". http://servicios2.abc.gov.ar/docentes/efemerides/10dediciembre/descargas/elecciones/asunci onpresi.pdf; para este tema ver además Romero (2006).

17 Esta afirmación se confirma por ejemplo en la versión de la historia conceptual encabezada por Giuseppe Duso y Sandro Chignola (2009).

18 Esta relación no es ninguna novedad. Es sabido que el proyecto liberal se basa en una concepción igualmente "científica" de la economía.

19 Esta modalidad del progreso es algo que el psicoanálisis ha trabajado intensamente en términos de postergación del goce (Žižek, 2002).
}

\section{anuario.}


hicieran ya "los modernos" frente a "los antiguos", que conocemos hoy más y mejor que ayer, pero seguramente menos que en el futuro.

Y aquí estamos.

Puede que muchos no estén de acuerdo con esta rápida descripción de la historia de la historiografia, y eso está bien, ya que sólo pretende señalar un punto: estas y otras muchas transformaciones teóricas, componen la memoria disciplinar de la historiografia. La historia teórica de la historiografia es inseparable de los debates historiográficos sobre eventos y procesos particulares producidos en y sobre su terreno, y produce teoría en su propia marcha, ya que supone una reexaminación constante de la validez y de la productividad de los principios que rigen dichos debates. Esto es, evalúa su pretensión de verdad.

A pesar de las muchas variaciones locales y preferencias personales, esta memoria se alimenta a todos los estudiantes universitarios que se especializan en historia. Lo que es más, esta tradición teórica común, con sus idas y venidas, y no un conjunto de procedimientos estandarizados que podrian considerarse específicos y diferenciarla de otras disciplinas, es lo que define nuestro trabajo como historia propiamente dicha, incluso si nuestros objetos de estudio a menudo están más relacionados con la política, la economía o la cultura, el arte o cualquier otra cosa: la identidad de la historiografia se construye históricamente. La forma histórica de comprensión se define por nuestra consideración y ponderación, ya sea con más o menos conciencia, de estas muchas definiciones de la historia. Por eso los historiadores profesionales no detentan la exclusividad de la interpretación histórica, que está presente en cualquier esfuerzo de interpretación que considere y actualice, en su interpretar, dicha memoria.

\section{La teoria de la historia es algo que hacemos}

Como disciplina establecida que se enseña en las universidades, estas teorías de la historia componen la estructura sobre la cual se espera que estudiantes y profesionales contribuyan con su investigación. La profesionalización iniciada en el siglo XIX significó el desarrollo de un código común para la comunidad de historiadores, apoyado en una red de revistas, más o menos prestigiosas, instituciones de financiación, cátedras universitarias y cursos, donde se organiza y articula el debate historiográfico. La globalización del código común para un campo trasnacional de la historiografía se desarrolló bajo la idea de la revisión por pares y del control de las interpretaciones

\section{anuario.}


aceptadas en subcampos cada vez más diversos, cada uno con sus propios métodos, temas, debates, lenguajes y conceptos. Hay un formato acordado que se debe dar a las tesis, artículos, libros, e incluso ensayos, si se pretende que se publiquen en los vehículos internacionalmente reconocidos para los debates de la disciplina, o que sean aceptados en conferencias y reuniones. Aunque así se presenta, este formato excede el objetivo de establecer un estándar de calidad para nuestro trabajo: expresa una concepción de la historia, un estado de la discusión y el lugar relativo que ocupan las diferentes ideas de lo que constituye el conocimiento histórico. Históricamente, el establecimiento y la delimitación del campo, y su globalización, han ido de la mano, definiendo unos parámetros más o menos comunes, que se reproducen tanto en los debates locales como en los internacionales ${ }^{20}$.

A través de estos mecanismos, la teoría de la historia - que también podría construir una memoria propia recurriendo a la veterana tradición de reflexión sobre la historia de innumerables pensadores - se constituyó en uno de los subcampos de la historiografia. Trata y enseña diferentes entendimientos y configuraciones de la tradición que describimos en el apartado anterior. Organiza herramientas de comprensión según su uso, significado, historia o adscripción politica y funciona a nivel normativo, proponiendo estándares para el conjunto de la disciplina ${ }^{21}$. Sin embargo, la mayoría de estas normas y estándares no son consideradas importantes por los historiadores que trabajan con la historia "propiamente dicha" (no teórica), por lo cual la teoria de la historia se ha convertido en algo que hacen los filósofos, no los historiadores ${ }^{22}$. Y este es un problema, no porque los teóricos sepan más que los "historiadores propiamente dichos", sino porque los dispositivos que se esfuerzan por hacer visibles, así como las normas que establecen, están en funcionamiento en cada trabajo historiográfico, no importa cuán objetivo, científico, aséptico o incluso políticamente correcto pretenda ser. Esto es, por más reparos que los historiadores puedan oponer a la teoria, no es posible articular una interpretación coherente respecto de un episodio determinado

20 La gran mayoría de las publicaciones académicas evalúan los artículos enviados en términos de su adecuación al paradigma científico, desconsiderando trabajos que no respondan a sus criterios y rechazando, por ejemplo, trabajos en formato de ensayo o reflexiones teóricas. Ver Lvovich (2009); Beigel y Bekerman (2019); Segato, (2015). En inglés, la presión por publicar en determinados medios se condensa en la expresión "publish or perish". Véase Moosa (2018). Sobre las evaluaciones de artículos enviados a publicaciones periódicas ver: http://theconversation.com/perish-not-publish-new-study-quantifies-the-lack-of-femaleauthors-in-scientific-journals-92999

21 Existe una variedad importante entre los trabajos de teoría de la historia, que van desde esfuerzos normativistas, como por ejemplo el de Jörn Rüsen (2013) hasta historizaciones descriptivas como las de Bourdé y Martín (1992), todas las cuales se postulan como visiones objetivas sobre la práctica histórica.

22 Basta recordar la enorme preponderancia de filósofos en las redes, congresos, comités evaluadores y publicaciones sobre teoría de la historia (como por ejemplo: https://www.inth.ugent.be) y la escasez de revistas sobre teoria de la historia (se destacan a nivel internacional History and Theory; Rethinking History; Storia della Storiografia; Historia da Historiografia; Historein y Journal of the Philosophy of History, cuyas páginas en general son pobladas por filósofos, y los pocos artículos de teoria que aparecen en las revistas de historia). 
sin movilizar presupuestos teóricos 23 . Y la teoría está en funcionamiento no solo a la hora en que el historiador se sienta a escribir, sino también en todos los mecanismos de construcción del campo, porque es lo que construye el argumento de verdad que los sostiene: el otorgamiento de financiamientos para la investigación, la evaluación de publicaciones, la construcción de cátedras en las universidades, la definición de áreas estratégicas, etc., dependen de que el argumento de verdad de la investigación en cuestión sea aceptado como válido.

En los últimos cincuenta años, la teoría de la historiografia ha experimentado una transformación fundamental, enriqueciendo sus discusiones mediante el acercamiento a diversas disciplinas, e inclusive cuestionando la división disciplinar tradicional, proponiendo generalizaciones teóricas que han sido denominadas "transdisciplinariedad", "interdisciplinariedad", "genealogia" o "globalización". Simultáneamente, se ha diversificado en campos cada vez más especializados, como la historia intelectual, la historia conceptual, la historia espacial y poscolonial, o los estudios de memoria, entre otros. Además de estos movimientos de contracción y expansión del campo específico, la profesión histórica en general ha experimentado discusiones teóricas transversales, a veces designadas "giros" 24 . Esta denominación hace referencia a discusiones enfocadas en diferentes problemáticas teóricas que modifican, o establecen criterios normativos según los cuales debería revisarse la práctica del historiador, como el giro espacial ${ }^{25}$, el giro moral ${ }^{26}$, el antropoceno ${ }^{27}$, el giro hermenéutico o el giro experiencial28. Más allá de las denominaciones particulares que pudiera asumir, la discusión actual parece dirimirse entre aquellos que proponen reconocer el carácter necesariamente político de la investigación y aquellos que sostienen la necesidad de retornar a un ideal de investigación objetiva ${ }^{29}$, ambos sobre el trasfondo de la sacudida producida en el campo de la producción de conocimiento por la secuencia de desastres históricos del último siglo.

\footnotetext{
23 El concepto que más explícitamente expresa esta afirmación es el de "entramado", propuesto por Hayden White (1992), que considera que es precisamente la puesta en trama lo que confiere verosimilitud a cualquier representación histórica. Paul Ricoeur (1983) dedicó los tres volúmenes de su Tiempo y narrativa a demostrar que la temporalidad, en tanto función articuladora del discurso, se construye en el momento de la narrativización. Pero la dependencia entre representación y teoría no se limita a los autores relacionados con el giro lingüístico. Por nombrar solo un ejemplo, Dipesh Chakrabarty (2000) muestra el peso de las teorias occidentales sobre las historias de los pueblos colonizados.

24 El primer "giro" es comunmente identificado con la publicación de Rorty (1967).

25 Para este tema, véase Usborne y Kümin (2013, pp. 305-18).

${ }^{26} \mathrm{Al}$ respecto, véase el texto Cotkin (2008, pp. 293-315).

27 Véase, por ejemplo, Palsson y Szerszynski (2013).

28 Aquí se encuentra encuadrada, por ejemplo, la discusión sobre el papel del testimonio. Véase Tozzi (2012).

29 Un libro importante recientemente publicado sobre el tema es Berger (2019).
} 
De modo que, desde la teoria, podemos organizar y analizar estas estructuras $\mathrm{y}$ dinámicas, rastreando nombres, instituciones y discusiones. Al hacerlo, tomamos posición respecto de la validez del argumento de verdad de cada propuesta. Esta valoración se realiza, principalmente, a través de la selección que hacemos de corrientes historiográficas significativas, y por medio de la forma en que las organizamos. Sin embargo, las valoraciones respecto de la validez y la efectividad de cada teoría no siempre llevan explícitos los presupuestos a partir de los cuales se realizan, situación que se da especialmente cuando la valoración se enmarca en el paradigma científico, que se supone aséptico y normativizado.

Veamos un ejemplo concreto. En 1972, el historiador Alberto Pla señalaba que las líneas representativas de la historiografia eran muy escasas, y destacaba la existencia de dos grandes corrientes en las cuales podía enmarcarse la mayor parte de los trabajos historiográficos. La primera era una historiografia "que estudia el acontecimiento en sí mismo y cuyo centro de estudio ha sido y es la historia politica, la epopeya del héroe nacional, los grandes hechos heroicos". La segunda se posicionaba en un tiempo largo braudeliano, y entendía la historia "como un conjunto de elementos (...) que deben ser armonizados en el lento cambio de las estructuras" (Plá, 1972: 9-11). La primera era denominada la "línea liberal de la historia tradicional"; y la segunda, historia económica o social, cuyo origen rastreaba Pla hasta el pensamiento marxista (Plá, 1972). A diferencia de la mayoría de los esfuerzos de clasificación, en los que las especificaciones sobre las preferencias del autor brillan por su ausencia, Pla explicita que la selección se realiza desde una perspectiva marxista, en una sociedad "desgarrada por la crisis social más impresionante de su historia", en la que el "manejo del pasado" responde tanto a cuestiones científicas como políticas.

Político o no, Pla no es el único historiador argentino de la época que adhirió a la corriente de la historia social. De hecho, podría decirse que tal corriente historiográfica acabó por convertirse en la ortodoxia en estas latitudes. Silvia Sigal, por caso, identifica para los años 60 una historiografia relacionada con la línea "Mayo-Caseros", que se opone a la historiografia de la "Renovación" posperonista (Siga, 1991), de corte netamente annalista, y abrazada por los historiadores más significativos de la época, como Emilio Ravignani, José Luis Romero o Tulio Halperin Donghi. A su vez, en la medida en que estos últimos historiadores, referencias del campo, articularon sus discursos historiográficos en el lenguaje de la historia social, y con ese mismo ideario fueron protagonistas del establecimiento institucional de la disciplina, la historia social pasó a ser relacionada directamente con la profesionalización de la historiografia (Miguez, 2006). Esa relación entre historia social y profesionalización, que se extiende y se reafirma con el retorno de la democracia en 1983, coloca a los métodos y objetivos teóricos de la historia social como el punto de comparación con el cual se evalúa la efectividad y la 
validez del resto de las teorias historiográficas en circulación. Al observar este debate más de cerca percibimos, sin embargo, que aunque se identifique a los historiadores sociales como opositores a la línea "Mayo-Caseros", y si bien hay una ampliación hacia aspectos económicos y sociales, lo que continúa explicándose es en definitiva el camino que llevó de Mayo a Caseros ${ }^{30}$. En este ejemplo, y a pesar de que las interpretaciones están más cercanas de lo que quisiera admitirse, la historia social aparece frecuentemente como el punto final de una evolución de la historiografia ${ }^{31}$, en vez de ser señalada como una corriente historiográfica entre otras. Pareciera que se confunde la denominación "historia social", que corresponde a un conjunto discreto de argumentos de verdad y de metodologías, con la "historia de la sociedad", esto es, con la ampliación del espectro de interés de la historiografia desde lo estrictamente político o heroico, al conjunto de la sociedad. Como una de las consecuencias de esta preponderancia (¿podríamos decir hegemonía?) teórica, podemos señalar el desprestigio, muchas veces acrítico, de las teorías a las cuales se opuso o se opone la historia social europea o vernácula, como por ejemplo el historicismo ${ }^{32}$, la hermenéutica o la historia filosófica, que se descartan como teorias demasiado antiguas, demasiado poco científicas o simplemente pasadas de moda.

Un buen ejemplo de un debate internacional que contribuyó a lo que más arriba identificamos como la globalización del campo es lo que algunos historiadores han llamado "el canon del Holocausto", algo que quisiera describir no como un conjunto de autores o temas ${ }^{33}$ sino, desde el punto de vista de la teoría, como una constelación de significados, que movilizan conceptos como la retórica del silencio, el mito de la Stunde Null, la dialéctica de los dos demonios, la alegoría del líder desquiciado y de la población cautiva y el trauma. Nacidos de la respuesta de los historiadores alemanes enfrentados al descubrimiento de los crimenes del nazismo, estos arquetipos parecen repetirse en la explicación o, mejor dicho, en el encuentro, con "pasados que no quieren pasar"34.

\footnotetext{
30 A eso se refiere la idea de una "estructura coralina" de la historiografia argentina (Adamovsky, Bisso y Di Meglio, 2011).

31 Es claro que esto no es un fenómeno local, pero basta repasar los textos que figuran en las bibliografias de la mayoría de los cursos introductorios o de historiografia que se dictan en las universidades argentinas. Por ejemplo, Moradiellos (2001); Fontana (2001); Iggers (2012). Esto también aparece en el mismo texto de Plá (1972), como se lee en la página 21:"el enfoque contemporáneo de la historia económica y social recupera la vieja historia erudita incorporando la erudición de ese conocimiento (...) en una explicación más completa, más compleja, y también más heterogénea..."

32 La publicación colectiva A Dinâmica Do Historicismo: Revisitando a Historiografia Moderna. (2008) refleja una nueva evaluación del historicismo que se desarrolló sobre todo en la década pasada.

33 Dicha expresión tiene, sin embargo, significados muy diversos. Véase Finchelstein (2010).

34 Para algunas respuestas a rupturas del lazo social que desafian las formas tradicionales de hacer historia, véase Bevernage (2014). Para el caso de Argentina, véase Feld y Franco (2015).
} 
Al igual que en el caso de la historiografia argentina de los años 60 que discutimos anteriormente, debemos comprender que tales arquetipos no son un mero asunto formal, pues no hay "asuntos meramente formales" en la historia, en la medida en que la forma de la mediación histórica es constitutiva del contenido mediado. Esto es, la mediación consiste en el montaje de una serie de correlaciones que conforman el argumento de verdad, y cuyo resultado es la indisociabilidad entre datos y argumento. La apropiación de estos conceptos tampoco es una respuesta por ventura natural a las rupturas violentas del vínculo social en general, como algunas teorias - la del trauma, por ejemplo - parecen sugerir. Estos arquetipos se toman expresamente, por vía de diversos procedimientos teórico-metodológicos - como la comparación o la conceptualización de la experiencia - como un conjunto de herramientas que se pueden extrapolar de su ámbito original, y traducir a experiencias locales. Dicho procedimiento implica, de por sí, una aceptación de los postulados de la historia científica ${ }^{35}$. Y si bien esto puede ser posible, e incluso productivo en algunos casos, importa reconocer que la extrapolación de una teoría sin su referente supone, en sí, una serie de decisiones teóricas y políticas diseñadas para lograr un cierto consenso en cuanto al significado de lo narrado, sea dentro de la comunidad historiográfica o en una comunidad más amplia. No es lo mismo, para poner un ejemplo muy simple, calificar a una interrupción del orden constitucional como "revolución" o como "coup d'Etat", a una insurgencia como "resistencia" o como "subversión", o al mandato de un grupo político al frente del Estado como "administración" o como "régimen". Estas denominaciones, que son conceptos teóricos que establecen sentidos y relaciones con otros eventos que también proporcionan significado, expresan los consensos políticos de la comunidad de intelectuales en la que son movilizadas. Para este caso particular, como ya hemos esbozado más arriba, cada narración historiográfica de una ruptura violenta del vínculo social parece venir acompañada de un retorno a la fe en la objetividad científica, una restitución de la linealidad del tiempo, de la organización de eventos en una oposición dualista y de la confianza en la concepción tradicional de la distancia histórica como hiato entre pasado y presente. Estos no son supuestos teóricos menores, y tienen consecuencias teóricas y políticas muy significativas para todos los involucrados en cualquier conflicto que, por cualquier razón, debe o no debe transformarse en pasado ${ }^{36}$.

Estos dos ejemplos permiten, por un lado, acercarnos a la idea de que las narrativas historiográficas requieren consenso politico con respecto a lo que

35 La mayoría de los manuales de escritura de trabajos científicos así lo establece. Véase, por ejemplo: Jablonska (2008, pp. 133-49). Como un ejemplo de la postura de la historia como ciencia, véase: Sánchez Jaramillo (2005, pp. 54-82). Esta afirmación debe asociarse, además, con la idea de la historia Hempeliana que citamos más arriba.

36 Uno de los mejores ejemplos de esta temática continúa siendo el artículo Nolte (2007). Este artículo desató la polémica sobre la necesidad de transformar al pasado en pasado. Asimismo, esta polémica ha cobrado fuerza siempre que se han propuesto amnistias para perpetradores de crimenes contra la humanidad. 
puede y no puede decirse, sea dentro de la comunidad de historiadores académicos o en el escenario más amplio de los sectores que tienen intereses en dichas narrativas ${ }^{37}$. Por otro, plantean la necesidad de una conciencia más profunda, por parte de los historiadores, de que es necesario considerar el impacto posible de sus afirmaciones, además de considerar su veracidad. Sin embargo, en la práctica y más allá de toda prudencia, en las construcciones teóricas que montamos al escribir, sucede como en el resto de las acciones de los hombres y mujeres en la historia, y como lo describiera Reinhart Koselleck (1993) con la frase "En la historia sucede siempre más o menos de lo que está contenido en los datos previos" (p. 248). Y esto me lleva al apartado final.

\section{Algo que sucede.}

"El objeto es la continuación del sujeto por otros medios"38

Una tercera dimensión de la teoría histórica se define mejor por una frase del filósofo Hans-Georg Gadamer (2005) en el prólogo a la segunda edición de su Verdad y Método. En ese trabajo, Gadamer definió su interés por la comprensión como "no lo que hacemos o lo que debemos hacer, sino lo que ocurre con nosotros por encima de nuestro querer y hacer" (p. 10). La afirmación de Gadamer significa que, en forma similar a la manera en que la filosofia kantiana describe la percepción del gusto (Kant, 1922), la comprensión precede a cualquier intento de aplicar sistemáticamente las herramientas para comprender que hemos adquirido y construido. En cierto modo, lo que nuestras herramientas y métodos nos permiten hacer es volver a describir, esto es, narrar nuestra comprensión, de una manera que pueda ser entendida y discutida por el resto de la comunidad. Ahora bien, si entendemos que no es posible ejercer un control absoluto sobre nuestras acciones de comprensión, como reza la frase de Gadamer, mal serviría al propósito de la verdad histórica una teoría de la historia que hiciera como si39 la indeterminación que habita la comprensión no produjese sentido.

Nuestra comprensión es así un elemento nuevo introducido en el mundo, que es comparable en muchos aspectos a la producción de una obra de arte y definitivamente diferente de una prueba científica. Al entrar en contacto con un fenómeno, interpretación o argumento nuevo, toda la constelación de

${ }^{37}$ La idea de lo político que se utiliza aquí no es una novedad, y se relaciona con la definición de Michel Foucault en términos de "la sociedad en la que vivimos, las relaciones económicas en las que funciona y el sistema que define las formas regulares, los permisos y las prohibiciones que rigen regularmente nuestra conducta". Citado en: Raffin (2018, pp. 29-59).

38 De Sousa Santos (2009).

39 El tratado más comprehensivo de la filosofia del "como si" es aun Vaihinger (1922). 
experiencias del historiador, que no se limita a lo que llamamos experiencia "personal" o "directa", sino que involucra sus lecturas, ideas de historia y tiempo, su opinión sobre la validez de la ciencia, los conceptos en los que se basa para entender el mundo presente, pasado y futuro, sus análisis politicos, sus proyectos, e incluso la literatura que lee, se ponen en juego simultáneamente en una única intuición (Gadamer, 2006). Esto es, cada situación, corresponda al orden temporal al que corresponda, se nos presenta como un todo, y no fibrilada en parcelas discretas: se trata de un movimiento mejor ilustrado por el momento en que un artista mira su pintura y decide que está terminada, aunque una vez alguien dijo que las obras de arte nunca se terminan, sino que se abandonan -y esto también es una buena descripción del trabajo del historiador, a riesgo de caer en el problema borgiano de la representación total ${ }^{40}$.

El resultado de este principio, que es el fundamento estético que Gadamer propone a los historiadores, es que la mediación operada por los historiadores no se limita a la introducción de conceptos, interpretaciones y de una estructura narrativa para dar forma a unos "hechos" previamente establecidos, obtenidos de una realidad supuestamente autónoma. En otras palabras, la mediación historiográfica no es una operación de aplicación que se cumpla en el momento de transmitir los resultados de la investigación. Más bien, cuando decimos que los historiadores dan sentido a la realidad, estamos diciendo que todo este complejo de dispositivos más o menos voluntarios, más o menos aprendidos, está en acción desde el comienzo de la investigación histórica. Los resultados que podemos producir en el camino de la investigación se modifican y adquieren nuevos matices en la medida en que reconocemos que este conjunto inicial se modifica y va trazando una dirección - que es otra acepción de la palabra "sentido" - para la investigación.

En esa misma línea, la concepción estética nos advierte que los historiadores no hablan sobre un pasado previamente definido, sino que en realidad negocian, proponen y definen continuamente la línea que delimita aquello que debe considerarse pasado, esto es, construyen un ordenamiento temporal, a partir de su perspectiva. Y si es cierto que no hablan sobre un pasado establecido a priori, también es cierto que hablan, en cambio, con el pasado a cuestas, es decir, cargados con la marca indeleble de sus convicciones adquiridas en el acto de definición de ese pasado. Esta marca permanece integrada en sus interpretaciones, de modo que es imposible separarla de la realidad que se narra, sin desmerecer por eso la seriedad y el cuidado metodológico que el historiador pueda desplegar. Considerando esta proposición, sería un error pensar solo la etapa de "presentación de resultados" como la fase de interpretación de la historiografia, que se apoya

40 Me refiero particularmente a los célebres "Funes, el memorioso" y "Del rigor en la ciencia".

\section{anuario.}


sobre un conjunto de hechos, estos sí, reales y objetivos ${ }^{41}$. Una teoría de este tipo olvidaría que la elección de un tema, su formulación y la selección y organización de "fuentes" y teorías para respaldar nuestro argumento también son producto de una comprensión de cómo son o deberían ser las cosas, de una mediación, que ya está en funcionamiento cuando algo aparece ante nosotros como un problema, como algo que requiere tematización. Sería ingenuo imaginar que, con el reconocimiento de la naturaleza constructiva de nuestro trabajo, viniera también la ilusión de poseer la capacidad de controlar todos los aspectos de la añadidura que el entramado (la organización en una trama) conlleva, pues reconocemos que el acto del entramado comienza mucho antes de expresar nuestros resultados en palabras ${ }^{42}$.

Este último fue el sueño de la objetividad que dominó el siglo $\mathrm{XX}^{43}$, $\mathrm{y}$ especialmente del subcampo de la historia social en tanto que se consideraba historia científica, por oposición a un historicismo meramente idiosincrático ${ }^{44}$. Fue, junto con la presuposición de la linealidad del tiempo, uno de los fundamentos de la permanencia de la idea del progreso del conocimiento, mediante la cual se depositó la fe en un futuro donde, a través de una investigación con mejores técnicas, más controlada metodológicamente y más profunda, la verdad finalmente se abriria paso, atravesando el medio (o el vehículo) del lenguaje y quedando expuesta en su transparencia, como lo describe el ángel - caído - de la historia de Walter Benjamin (1973) ${ }^{45}$.

Uno de los dispositivos más eficaces de que se ha servido el paradigma científico para cimentar su hegemonía consiste en negar la existencia de una

41 Hayden White (2000) estableció una distinción entre "hechos", que serían conjuntos de acontecimientos establecidos como históricamente relevantes, y "acontecimientos", los cuales corresponderían a algo así como "la cosa en sî" kantiana, que es incognoscible por definición. Con esta distinción, White pretendia concentrarse en la narrativa histórica, sin entrar en el problema de la veracidad de lo narrado. El problema con esta distinción es que en rigor no existe ningún motivo para considerar que el establecimiento de los "hechos" no posea una carga teórica en su propia construcción como tales. De otro modo, ¿de acuerdo a qué criterios, sino criterios teóricos, se hubiera podido establecer su relevancia histórica?

42 Este es el punto en el que la concepción estética de la filosofia hermenéutica gadameriana difiere de la teoría constructivista de la historia: mientras que esta última separa la investigación de la escritura (véase nota anterior) la concepción estética entiende que el fenómeno de la comprensión involucra todo el proceso. Para un repaso de la teoría constructivista véase Pihlainen (1998).

43 Para una historia de la objetividad en los últimos siglos, véase Daston y Galison (1992)

44 Para el argumento de la cientificidad de la historia en la historia social, los textos de Chartier (2005) resultan los más ampliamente aceptados entre los historiadores.

45 La tesis de Benjamin (1973), que ha servido para sustentar posturas muchas veces contrapuestas, quiere aquí recordarnos la imagen de un tiempo que en su desarrollarse revela progresiva y automáticamente la verdad que estaba antes oculta tras el velo del interés inmediato, coyuntural, del cual podríamos despegarnos al alejarnos de los eventos que nos ocupan. Esta es la concepción que subyace a la tesis "generacional": aquella que supone que solo una generación posterior a aquella que ha protagonizado determinados eventos posee la distancia necesaria para criticarlos objetivamente.

\section{anuario.}


discusión entre diferentes formas de la verdad. Boaventura de Sousa Santos (2009) se refiere a esta forma de pensar el conocimiento como "abismal", y afirma: "En el campo del conocimiento, el pensamiento abismal consiste en conceder a la ciencia moderna el monopolio de la distinción universal entre lo verdadero $\mathrm{y}$ lo falso, en detrimento de dos cuerpos alternativos de conocimiento: la filosofia y la teologia" (p. 162). Se trata del mecanismo que De Sousa define en otro lugar como "epistemicidio", que consiste en negar entidad a cualquier forma no-científica de la verdad.

Dentro de esta idea general de De Sousa, propongo substituir a la teología por la estética, la cual, entendida en su amplitud filosófica, engloba al pensamiento y a la verdad religiosa ${ }^{46}$, y tiene la virtud de poner de manifiesto existencias que se encuentran allende la línea que el paradigma científico traza entre lo verdadero y lo falso. Esto quiere decir que, además de la "verdad revelada", que en algunos casos sí ha sido parte de la verdad aceptada dentro de las fronteras del paradigma científico, ${ }^{47}$ la estética - en los términos planteados al inicio de esta sección - nos permite el acceso, por ejemplo, a la verdad sentida, intuida ${ }^{48}$ y creada; abre las puertas a la verdad de lo sublime, en su acepción de aquello que excede la capacidad del entendimiento conceptual (Kant, 1922) ${ }^{49}$.

La estética, sin embargo, no goza de buena reputación entre los historiadores científicos ${ }^{50}$, y suele ser identificada con su opuesto complementario en el mejor de los casos, esto es, como lo que recae en el orden de la invención, del embellecimiento o de la ornamentación, cuando no con el significado más pueril y despectivo del concepto de retórica, esto es, de la argumentación maliciosamente engañosa. El concepto de lo estético que intentamos movilizar aquí apela, por el contrario, a la capacidad creativa que se despliega al producir una interpretación a partir de elementos percibidos, o al aprehenderla. Se trata de esa facultad que Immanuel Kant identificara tradicionalmente con la imaginación, aunque aquí propongamos, además, volver a reunir a la imaginación reproductiva y a la imaginación productiva que este filósofo tan hábilmente separara51. De hecho, quisiéramos proponer que el "epistemicidio" antes mencionado constituye un producto de esta

\footnotetext{
46 Nuevamente aquí puede ayudarnos Gadamer (2006).

47 Sin ir más lejos, en el argumento del Dios engañador del Discurso cartesiano.

$48 \mathrm{La}$ intuición aquí tiene un sentido más amplio que el de adivinación. Intuición remite a la puesta en funcionamiento de todo el conjunto de experiencias que intervienen en la percepción de un fenómeno. La intuición es "una cualidad especial de la descripción o de la narración, de tal manera que lo que uno no ve él mismo, sino que solo se lo cuentan, llega a verlo, por así decirlo 'delante suyo". Esta es, en definitiva, una cualidad estética”. Gadamer (2006., pp. 153172, p. 154).

49 Hans Blumenberg (2003) ha propuesto otra forma de aproximarse al trabajo de interpretación que critica la hegemonía del ideal de la conceptualidad.

50 Ver, por ejemplo, el uso de la expresión “contemplación estetizante” por parte de Ginzburg (1981, p. 7).

${ }^{51}$ Hemos propuesto una discusión de esta operación en Gay (2015).
}

\section{anuario.}


división entre "reproducción" y "creación", que traza la frontera última entre el conocimiento científico, (o la episteme) y la tierra sin ley de la doxa.

De Sousa (2009) ayuda nuevamente a comprender este mecanismo al describir el caso de las fronteras establecidas por las autoridades nacionales, que delimitan un espacio de aplicación del derecho, fuera del cual rigen normas diferentes, o no rige ninguna. El otro lado de la frontera o la propia zona de frontera, suele ser no solo el territorio del desorden, sino también del abuso de autoridad, porque se encuentra fuera de, o en el limbo del espacio de aplicación de la ley y, por lo tanto, del castigo a los abusos. Como con las fronteras espaciales sucede con el conocimiento: se supone que aquello que se encuentra más allá de la frontera de lo que es "científicamente cognoscible" recae en la categoría del "todo vale". Esta ha sido la reductio ad absurdum con la que se ha descartado todo tipo de verdad histórica que no se pretenda científica, pues se le adscribe un relativismo absoluto, con la consecuente carencia de valor de verdad. Asimismo, la cientificidad del historiador se mide, en general, según su apego a los documentos. Pero si el trabajo del historiador no es previsible - esto es, definible a priori -, ni genera resultados universalizables - esto es, la posibilidad de extrapolar una explicación para aplicarla a situaciones similares -, ni reproducibles - esto es, pasibles de ser repetidos para comprobar que el resultado es el mismo -, si todos los datos son opacos, manipulables, parciales y en consecuencia necesitados de interpretación heurística; si las interpretaciones sobre los mismos documentos pueden muchas veces ser diametralmente opuestas, entonces la verdad histórica debe descansar en otro lugar que no es la evidencia documental, a pesar de que ésta sea fundamental para construirla, en la medida en que conforma nuestro universo tradicional de sentido.

De acuerdo con lo que se ha dicho anteriormente, me gustaría proponer que la verdad última de la historia se asienta sobre una experiencia diversa y particular, pero compartida. La experiencia, sin embargo, aparece como otro concepto problemático para el paradigma científico ${ }^{52}$, desde el cual también se ve separada por una frontera. De un lado, aparece la experiencia que es sinónimo de experimento, que es preparada a partir de protocolos regulados, y que produce resultados que se consideran epistemológicamente válidos. Del otro, la experiencia como dato "puro" de la realidad, que se identifica con lo personal, carece de la verificación necesaria y por lo tanto se considera

52 La experiencia tiene su propia historia. Para la teoría de la historia, existen algunos clásicos del tema, como Dilthey (1985); Oakeshott (1933); Scott (1991) (esta última muy importante en el debate sobre el marxismo culturalista británico); Agamben (2011), entre muchos otros. Para un panorama histórico de la discusión es fundamental el libro de Jay (2009). Recientemente, se ha retomado la discusión sobre el concepto desde perspectivas alternativas. Ver, por ejemplo, Carr (2014). 
subjetiva y parcial. Esta división, como otras que hemos señalado, prefigura un sentido de la interpretación que obtura otros significados posibles. Por eso, no queremos rehabilitar aquí la experiencia como experimento, que no tiene lugar en disciplinas como la historiografia, pues como ya hemos establecido, este procedimiento resulta imposible de implementar. Tampoco se trata de la experiencia de los hechos en sí mismos, que sí posee más presencia en las formulaciones teóricas historiográficas, porque sabemos muy bien que, incluso al observar la misma escena actual, las interpretaciones de los observadores variarán inevitablemente ${ }^{53}$. Por lo tanto, no proponemos una especie de verdad consensual, ya que, entre otros problemas, la verdad consensual implicaría la equivalencia de las percepciones de aquellos que participan en el debate (a la Habermas) ${ }^{54}$, o el supuesto de una agregación acumulativa de perspectivas.

Por fuera de estas divisiones clasificatorias, pensamos en la experiencia que se constituye al leer, ver, votar y estudiar, además de caminar en el mundo: de formar parte de una tradición con la que podemos estar de acuerdo o en desacuerdo en diferentes momentos, en situaciones específicas y en diversos niveles. Esta experiencia es definida por la tradición humanística como formación ${ }^{55}$, y describe tanto un proceso - el formarse - como un estado - la percepción actual del mundo en función de la experiencia de formación presente. La tradición compone el horizonte de sentido dentro del cual podemos pensar el mundo ${ }^{56}$. Por supuesto, la apropiación de, y la disputa por la tradición no es igual para un académico de la ciudad más rica de Europa o para el profesor de una universidad africana, para un profesor universitario o para un estudiante de primer año de la universidad, o para una mujer. Los "hechos" aparecerán bajo luces muy diferentes según el lugar desde dónde estamos leyendo y, en tanto que realicemos una lectura propiamente histórica de la teoria, debemos considerar esta alteridad cuando juzgamos la verdad propia de las posiciones teóricas propias y ajenas. Esto no significa que podamos afirmar cualquier cosa. En cierto modo, significa todo lo contrario: significa que es un error separar el mundo vivido del mundo aprendido, esto es, dividir la experiencia de descubrir el mundo en función del soporte en que se presenta.

\footnotetext{
53 Una vez más, vale recordar otro aspecto de la discusión sobre el valor del testimonio del testigo directo en la historiografia, que en ocasiones parece sugerir que solo quien ha atravesado personalmente ciertas experiencias es capaz de narrarlas con alguna autoridad. Aquí puede ayudarnos nuevamente De sousa Santos (2009).

$54 \mathrm{Me}$ refiero aquí a las críticas que Habermas ha recibido por causa de su teoría de la acción comunicativa, que supone un universo ideal de construcción del discurso democrático. Para un repaso de esta crítica ver Jaramillo (2010).

55 Gadamer define el concepto de formación en sus varios aspectos en la primera parte de Verdad y método (pp.38-48).

56 Para una definición del concepto de tradición, ver Bruns (1999). Para una narración que muestra la naturaleza polisémica y política de su utlización, ver Kleinberg (2012).
}

\section{anuario.}


El hecho de que ciertos argumentos se sostengan incluso en estas condiciones tan improbables, incluso si se producen fuera de los estándares académicos, es una prueba más de que la verdad histórica, que nunca es estable, nunca es indiscutible, nunca es una "cosa", no es equivalente a la "correspondencia con los hechos". Y eso no la convierte en una verdad menor. La correspondencia con los hechos expresa una concepción teórica particular de la historia y del conocimiento (la de la ciencia) ${ }^{57} \mathrm{y}$, por lo tanto, no debe asumirse como el criterio universal, como la frontera a partir de la cual puede juzgarse el trabajo historiográfico. Tampoco puede funcionar como una base que deba establecerse para poder - sólo entonces - proporcionar una interpretación, ya que, como el más famoso de los filósofos alemanes dijo una vez, es imposible decir que algo es, sin decir lo que es (Heidegger, 2000). Aquí nuevamente, la interpretación, desde un lugar de la tradición, ya ha tenido lugar: al representar - al mediar -, seleccionamos (al menos) la relevancia relativa, la escala, el orden y el ritmo de los fenómenos que, en un círculo virtuoso, se conforman mediante tales decisiones. Utilizando las palabras de Frank Ankersmit (2010): "la interpretación involucra no solo el establecimiento de un significado, sino también de un mundo" (p. 33) 58 .

Siendo así, la verdad histórica debe ser, de una vez por todas, disociada de otros atributos trascendentales, como el bien o la belleza. Dicha asociación hace que sea dificil entender la verdad como algo que no sea "verdad por correspondencia", y por lo tanto, como un extremo de dos opuestos (verdadero-falso; bien-mal; amigo-enemigo; sujeto-objeto; creatividadconocimiento) ${ }^{59}$. La verdad por correspondencia debe reconocer de una vez por todas que recoge del mundo solo lo que ella misma pone en el mundo, estableciendo así una frontera insoslayable.

Hemos intentado hasta aquí, proponer que la verdad en la historia designa un fenómeno diferente, cuya naturaleza es estética, y para cuya realización parece más conveniente apoyarse en la concepción hermenéutica, y sobre todo en su rehabilitación de la idea de "sentido común" (Gadamer, 2005). Si logramos librarnos de caracterizaciones binarias, la verdad histórica se puede definir como aquello con lo que nosotros (historiadores o no) podemos concordar, contribuir y disentir temporariamente, porque lo reconocemos como parte de nuestra propia experiencia del mundo (aprendido, leído, visto, entendido, caminado, deseado o temido). La verdad es aquello que permite que

\footnotetext{
57 Para esta discusión, continúa siendo de gran ayuda Dray (1957).

$58 \mathrm{El}$ énfasis es del autor.

59 La tensión entre creación y conocimiento fue traducida epistemológicamente en la dualidad de dos formas de conocimiento, ambas dependientes de las bases del paradigma científico moderno: el conocimiento aplicado (mecánico), y el conocimiento teórico (puro), división que limita las posibilidades de comprensión de la historiografia.
} 
la conversación continúe, se diversifique, evolucione y agregue significado al mundo que decimos como historiadores y como comunidad ${ }^{60}$. En este sentido, el pensamiento comunitario es algo diferente de la intersubjetividad, que es un concepto que sugiere un pacto o acuerdo entre individuos previamente definidos o entre disciplinas discretas. La comunidad significa que a medida que nos reconocemos a nosotros mismos y a nuestra formación, y nos sentimos interpelados por el debate en curso, construimos una tradición común: no nos sumamos a una historia o a un pasado previamente determinados, aun para discordar, sino que nos comprometemos en el esfuerzo común de la mediación. O, como decía Walter Benjamin (1989), "En toda época ha de intentarse arrancar la tradición al respectivo conformismo que está a punto de subyugarla” (p. 180). En esta formulación, la distancia histórica adquiere densidad a medida que reconocemos las diferencias entre el "nosotros" comunitario y aquello que consideramos pasado. Abríamos este ensayo diciendo que el mundo en el que Marc Bloch escribió su apología de la historia ha desaparecido, y esta sensación es la definición más adecuada de la distancia histórica; el pasado es aquello que podemos definir exitosamente como pasado, y los límites de este éxito son los límites de la comunidad.

La "inverdad", si hubiera un opuesto, sería aquello que detiene el diálogo, algo que ni siquiera provoca revuelta o disgusto, y por lo tanto no convoca al juego: ¿Cómo podrian entender los aymaras que la historiografia haya clasificado a su historia como "prehistoria" solo porque sus registros funcionaban con imágenes en lugar de palabras? ${ }^{61}$ No lo hacen. Ellos simplemente no son convocados a formar parte de la comunidad histórica occidental. La inverdad se reconoce inmediatamente, como el gusto, porque no exhorta a aquellos que no concuerdan con el relato transmitido a hacer oír su voz, no interpela a los agentes en su identidad, de modo que encuentren que vale la pena disputar dentro de la comunidad. Restringe, diria quizás Walter Benjamin (1977), "la comunidad de los que tienen el oído atento" (p. 446). Más allá de las incontables discusiones que este texto ha generado, interesa aquí recuperar la noción de experiencia de Walter Benjamin en su texto "El narrador", en donde se contrapone a la narración (cuya referencia es Heródoto) con la información. Lo que quisiera destacar de esa diferencia es que la primera comprehende al narrador en un modo que escapa a la diferenciación entre objetividad y subjetividad que es tradicional en la ciencia, y que expresa el sentido de comunidad más propio de la tradición humanística.

Esta verdad, la verdad práctica, si se me permite, que la historia puede proporcionar, es lo que explica el atractivo de la historiografia como un

60 En un artículo reciente, Hans Kellner (2019) definió a los clásicos como aquellas intervenciones que permanecen en la conversación luego de que su proponente ha desaparecido. En este sentido, diría que los clásicos son aquellos trabajos que contienen verdad.

61 Ver para esta reflexión: Condori (1992).

\section{anuario.}


instrumento para forjar la comunidad, incluso después de la ruptura más atroz del vínculo social: lo que la narrativa histórica restituye no es el pasado, sino el lazo comunitario. De allí la asociación "tradicional" de la historiografia con la nación, y también con la conformación de comunidades políticas alternativas, que destacábamos más arriba, y que funciona más allá del querer y el hacer de los historiadores e historiadoras profesionales. Y a la vez este poder de construir una comunidad, de pensar juntos como comunidad, señala la responsabilidad social y política que conlleva la creación de argumentos históricos, esto es, la mediación del sentido común - o mejor dicho, comunitario.

Así las cosas, podemos comprender que los historiadores del campo de la teoría de la historia no hacemos nada diferente de lo que hacen otros historiadores "propiamente dichos": buscar la verdad. Como historiadores posposmodernos, sin embargo, debemos tomar en serio, y tal vez fomentar, la disolución de la representación binaria del mundo y de los ideales de progreso y objetividad, rediscutiendo la noción de verdad que les subyace. De lo contrario, corremos el riesgo de reproducir una descripción del mundo que transforma no solo su pasado sino también su presente y futuro, en aquello que hemos descripto, como una profecía autocumplida en la que se oponen - y se opondrán necesariamente - sujetos y objetos, así como amigos y enemigos ${ }^{62}$. Cabe preguntarse finalmente si la articulación de la comunidad en estos términos - de dentro y fuera - no implica dar continuidad a la configuración del universo de sentido que dio lugar a las rupturas más dañinas del vínculo social en la historia.

Creo que esta tercera dimensión de la teoría de la historia es lo que la hace indispensable para la profesión historiográfica en su conjunto, dado que se nos ha otorgado la autoridad que viene con la institucionalización y la profesionalización. Como historiadores "propiamente dichos" debemos hacer teoría. Debemos analizar, discutir y también juzgar las consecuencias de lo que sabemos, lo que hacemos y lo que sucede mientras lo hacemos. Si estamos a la altura del desafio, decidiremos el futuro de la historiografia y, por qué no, el tipo de comunidad que construyamos.

62 Esta es la concepción schmittiana de la historia que aparece reproducida, por ejemplo, en los trabajos de Reinhart Koselleck (2012). 


\section{Bibliografia}

Adamovsky, E.; Bisso, A.; Di Meglio, G. (2011). ¿Hay nuevos relatos históricos para la Argentina actual? Sociohistórica, (30). Disponible en: http:/ / www.sociohistorica.fahce.unlp.edu.ar/article/view/SHn30a07/2457. Fecha de acceso: 05/10/2015.

Agamben, G. (2011). Infancia e Historia. Destrucción de La Experiencia y Origen de La Historia. Buenos Aires: Adriana Hidalgo Editores.

Ankersmit, F. (2012). Meaning, Truth, and Reference in Historical Representation. Ithaca: Cornell University Press.

Ankersmit, F. (1996). La Verdad En La Literatura y La Historia. En La Nueva Historia Cultural La Influencia Del Posteestructuralismo y El Auge de La Intersiciplinariedad. Madrid: Complutense, 1996.

Assmann, A. (2006). History, Memory, and the Genre of Testimony. Poetics Today, 27(2), pp. 261-273.

Beiser, F. (2011). The German Historicist Tradition. Oxford: Oxford University Press.

Benjamin, W. (1989). Discursos interrumpidos I. Filosofía del arte y de la historia. Madrid: Taurus.

Benjamin, W. (1977). Gesammelte Schriften. Frankfurt am Main: Suhrkamp Verlag.

Benjamin, W. (1973). Tesis de filosofia de la historia. Madrid: Taurus.

Berger, S. y Lorenz, C. (2010). The Contested Nation. Houndsmills: Palgrave Macmillan.

Bevernage, B. (2014). Historia, memoria y violencia estatal. Tiempo y justicia. Buenos Aires: Prometeo.

Bloch, M. (1996). Apología de la historia o el oficio del historiador. México: Fondo de Cultura Económica.

Blumenberg, H. (2003). Paradigmas Para Una Metaforologia. Madrid: Minima Trotta.

Braudel, F. (1970). La Historia y Las Ciencias Sociales. Madrid: Alianza Editorial. 
Bruns, G. (1999). What Is Tradition? New Literary History 22 (1): pp. 1-21.

Bury, J. B. (1920). The Idea of Progress. An Inquiry into Its Origin and Growth. London: Macmillan and Co.

Carr, D. (2014). Experience and History. Phenomenological Perspectives on the Historical World. Oxford: Oxford University Press.

Caimari, L (2017). La Vida En El Archivo. Goces, Tedios y Desvios En El Oficio de La Historia. Buenos Aires: Siglo XXI.

Chakrabarty, D. (2000) Provincializing Europe: Postcolonial Thought and Historical Difference. Postcolonial Thought and Historical Difference. Princeton: Princeton University Press.

Chartier, R. (2005). El mundo como representación. Estudios sobre historia cultural. Barcelona: Editorial Gedisa.

Condori, C. M. (1992). Los aymaras frente a la historia: Dos ensayos metodologicos. Aruwiyiri Chukiyawu.

Cotkin, G. (2008). History's Moral Turn. Journal of the History of Ideas 69 (2), pp. 293-315.

Croce, B. (1921). Theory and History of Historiography. London: George G. Harrap \& Co. Ltd.

Daston, L. y Galison, P. (1992). The Image of Objectivity. Representations 40, pp. 81-128.

De Sousa Santos, B. (2009). Una epistemología del sur. La reinvención del conocimiento y la emancipación social. México: Siglo XXI; CLACSO.

Devoto, F. (2006) (ed.), La historiografía argentina en el siglo XX. Buenos Aires: Editores de América Latina.

Dilthey, W. (1985). Poetry and Experience. New Jersey: Princeton University Press.

Dray, W. (1957). Laws and explanations in history. Oxford: Oxford University Press.

Duso, G. y Chignola, S. (2009). Historia de los Conceptos y Filosofía Política. Madrid: Bilbioteca Nueva. 
Feld, C. y Franco, M. (2015). Democracia hora cero. Actores, politicas y debates en los inicios de la posdictadura. Buenos Aires: Fondo de Cultura Económica.

Finchelstein, F. (2010). El canon del holocausto. Buenos Aires: Prometeo.

Fontana, J. (2001). La historia de los hombres. Barcelona: Crítica.

Hempel, C. (1942). The Function of General Laws in History. The Journal of Philosophy 39 (2).

Gadamer, H. (2006). Estética y hermenéutica. Madrid: Tecnos, (3 ed.).

Gadamer, H. (2005). Verdad y método. Salamanca: Sígueme.

Gay, M.E. (2015). Para um conceito moderno de imaginação: a imaginação do ponto de vista kantiano. História Da Historiografia, (18), 75.

Ginzburg, C. (1981). El queso y los gusanos. Barcelona: Muchnik Editores.

Hegel, G.W.F. (2005). Filosofia de La Historia. Buenos Aires: Claridad.

Heidegger, M. (2000). Ontología, la hermenéutica de la facticidad. Madrid: Alianza Editorial.

Herder, J.G. (1877). Vom Erkennen und Empfinden der menschlichen Seele. Sämtliche Werke, 33 vols., vol. 8. Berlin: B. Suphan y C. Redlich.

Herder, J.G. (2007). Filosofia de La Historia Para La Educación de La Humanidad. Buenos Aires: Nova.

Iggers, G. (2012). La historiografia en el siglo XX. Desde la objetividad científica al desafio posmoderno. Santiago de Chile: Fondo de Cultura Económica.

Jablonska, A. (2008). La Elaboración Del Marco Teórico versus La Ilusión Del Saber Inmediato Estudios. Estudios Sobre Las Culturas Contemporáneas XIV, pp. 133-49.

Jaramillo Marín, J. (2010). El Espacio de Lo Político En Habermas. Alcances y Límites de Las Nociones de Esfera Pública y Política Deliberativa. Jurídicas 7 (1), pp. 55-73.

Jay, M. (2009). Cantos de Experiencia. Variaciones Modernas Sobre Un Tema Universal. 1 ed. Buenos Aires: Paidós.

Jelin, E. (2003). Los derechos humanos y la memoria de la violencia política y la represión: la construcción de un campo nuevo en las ciencias sociales. Cuadernos Del Ides, 2. 
Kant, I. (2009). Crítica de La Razón Pura. 2da ed. Buenos Aires: Colihue.

Kant, I. (1922). Kritik der Urteilskraft. Leipzig: Felix Meiner.

Kellner, H. (2019). Durable goods. History and theory, v. 57, Issue S1.

Kleinberg, E. (2012). Introduction: The 'Trojan Horse' of Tradition. History and Theory 51 (4), pp.1-5.

Koselleck, R. (1993). Futuro Pasado. Para una semántica de los tiempos históricos. Barcelona: Paidós.

Koselleck, R. (1994). Vergangene Zukunft. Frankfurt am Main: Suhrkamp Verlag.

Koselleck, R. (2010). Historia/Historia. 2 ed. Madrid: Minima Trotta.

Kuukkanen, J. (2015). Postnarrativist Philosophy of Historiography. New York: Palgrave Macmillan.

Leuridan, B. y F. (2012). On Lawfulness in History and Historiography. History and Theory 51 (2), pp. 172-92.

Lopes de Araujo, V. (2008). A Dinâmica Do Historicismo: Revisitando a Historiografia Moderna. Belo Horizonte: Argumentum.

Lvovich, D. (2009). Resultados e Impactos de Los Programas de Apoyo a La Formación de Posgrado En Argentina. Revista CTS 13 (5).

Misak, C.J. (1995). Verificationism. Its History and Prospects. London: Routledge.

Moradiellos, E. (2001). Las caras de Clío. Barcelona: Grijalbo.

Mudrovcic, M.I. (2007). El Debate En Torno a La Representación de Acontecimientos Limite Del Pasado Reciente: Alcances Del Testimonio Como Fuente. Diánoia 52 (59).

Nolte, E. (2007). Un Pasado Que No Quiere Pasar. Pasajes: Revista de Pensamiento Contemporáneo, no. 24, pp. 71-75.

Oakeshott, M. (1933). Experience and Its Modes. Cambridge: Cambridge University Press. 
Olsen, N. (2012). History in the Plural: An Introduction to the Work of Reinhart Koselleck. United States: Berghahn books.

Palsson, G. y Szerszynski, B. (2013). Reconceptualizing the 'Anthropos' in the Anthropocene: Integrating the Social Sciences and Humanities in Global Environmental Change Research. Environmental Science and Policy.

Pihlainen, K. (1998). Narrative objectivity versus fiction: On the ontology of historical narratives. Rethinking History, 2(1).

Plá, A. (1972). Ideología y método en la historiografía argentina. Buenos Aires: Nueva Visión.

Raffin, M. (2018). La Noción de Política En La Filosofia de Michel Foucault. Hermenéutica Intercultural 29, pp. 29-59.

Raka R. y Radhakrishnan, S. (2010). The Subaltern, the Postcolonial, and Cultural Sociology. Handbook of Cultural Sociology.

Ranke, L. (1979). Pueblos y Estados En La Historia. México: Fondo de Cultura Económica.

Rendueles, C. (2013). Teoría Social y Experiencia Histórica. La Polémica Entre E. P. Thompson y Louis Althusser. Sociologia Historica 3, pp.177-97.

Ricoeur, P. (1983). Temps et Récit. 1 ed. Paris: Éditions du Seuil.

Romero, L.A. (2006). La Democracia y La Sombra Del Proceso. En Quiroga y Tcach (eds.) Argentina 1976-2006. Argentina Entre La Sombra Del Proceso y El Futuro de La Democracia. Rosario: Homo Sapiens.

Rorty, R. (1967). The Linguistic Turn. Essays in Philosophical Method. Chicago: The University of Chicago Press.

Sánchez Jaramillo, F. (2005). La Historia Como Ciencia. Revista Latinoamericana de Estudios Educativos 1, pp. 54-82.

Segato, R. (2015). Geopolítica del conocimiento: Universidad y país. Algunos ejemplos desde el Brasil. Disponible en: https://www.academia.edu/25253037/Geopol\%C3\%ADtica_del_conocimiento Universidad_y_pa\%C3\%ADs._Algunos_ejemplos_desde_el_Brasil .

Scott, J. (2010). Género e Historia. Translated by Consol Vila I Boadas. México: Fondo de Cultura Económica.

Scott, J. (1991). The Evidence of Experience. Critical Inquiry 17 (4): pp.773-97. 
Sigal S. (1991). Intelectuales Y Poder En La Década Del Sesenta. Buenos Aires: Puntosur.

Tilmans, K., van Vree, F., y Winter, J. (eds) (2010). Performing the Past Memory, History, and Identity in Modern Europe. Amsterdam: Amsterdam University Press.

Usborne, C. y Kümin, B. (2013). At Home and in the Workplace: A Historical Introduction to the 'Spatial Turn.' History and Theory 52, pp. 305-18.

Vaihinger, H. (1922). Die Philosophie Des Als Ob. Leipzig: Verlaf von Felix Meiner.

Vico, G. (1995 (1744)). Ciencia Nueva. Madrid: Tecnos.

Wallace, R. (1981). Progress, Secularization and Modernity: The LöwithBlumenberg Debate. New German Critique 22: 63-79.

White, H. (1973). Metahistory. The Historical Imagination in Nineteenth-Century Europe. Baltimore: The John Hopkins University press.

White, H. (2000). An Old Question Raised Again: Is Historiography Art or Science? (Response to Iggers). Rethinking History 4 (3).

Žižek, S. (2002). Enjoy Your Symptom. Jacques Lacan in Hollywood and out. (Vol. 111). New York: Routledge.

Recibido: 3 de marzo de 2020 